\title{
STATES, STATE TYPES, AND THE CAUSATION OF BEHAVIOR ${ }^{1}$
}

1.

In recent years it has often been argued that, although the relata of causal relations may well be events, not all properties of, say, a cause event are causally relevant in the same way for the production of the effect event. ${ }^{2}$ Especially in the context of the anomalous monism developed by D. Davidson this kind of argument has started off fresh discussions, particularly discussions concerning the question whether the mental has any genuine causal effect on the course of events at all or whether it is no more than an epiphenomenon. The reason is obvious, for Davidson's anomalous monism is characterized by the following three theses:

(1) Mental events causally interact with physical events.

(2) Event $c$ causes event $e$ only if there is a strict causal law which subsumes $c$ and $e$.

(3) There are no strict psychophysical laws. ${ }^{3}$

Davidson resolves the seeming inconsistency of these three theses by adding a fourth claim:

(4) Every mental event is a physical event.

If this claim is true a mental event $m$, despite the fact that there are no strict psychophysical laws, can cause a physical event $p$ if there is a physical law which subsumes both $m$ and $p$ under appropriate physical descriptions. But if psychophysical causation always rests on the existence of physical laws the mental properties of mental causes seem to be entirely irrelevant from a causal point of view. Whenever the mental event $m$ causes the physical event $p$ the mental properties of $m$ do not count, since if there is an appropriate physical law $m$ would cause $p$ only in virtue of the physical properties mentioned in this law, even if $m$ had no mental properties at all. So, on Davidson's account the mental seems to be entirely causally inert.

These considerations certainly highlight a very intriguing problem,

Erkenntnis 36: 267-282, 1992.

(C) 1992 Kluwer Academic Publishers. Printed in the Netherlands. 
though phrasing them in terms of the properties of events is, in my view, somewhat misleading. The properties which are decisive here are the properties of the things involved in the respective events. Let us consider an example: ${ }^{4}$ a haystack catches fire from a burning cigarette that has carelessly been thrown into it. Here the effect consists in the haystack catching fire and the cause in the throwing away of a still burning cigarette. The main objects involved, then, are the cigarette and the haystack. Obviously not all the properties of these objects are causally relevant for the effect event. Let us concentrate on the cigarette. Which of its properties are causally relevant and which are not? Most of them certainly are not. The color of the cigarette does not play any causal role, nor does its size or taste. What really matters is that the cigarette is still burning or, to put it in more general terms, that the cigarette has a certain temperature. Besides this property the only thing that counts is the fact that the cigarette is brought into contact with the haystack, i.e., the specific spatial relation between the cigarette and the haystack which is the terminus of the trajectory of the cigarette, which in turn is caused by the way it has been thrown.

How are we to reformulate the problem of the ineffectiveness of mental properties in terms of these considerations? This, in fact, is easily done. Most of us are convinced that, at least sometimes, we do something because we have certain wants and beliefs, i.e., that at least sometimes our wants and beliefs are the causes of our behavior. Such wants and beliefs are ordinarily construed as special kinds of mental states. So, wanting that $p$ and believing that $q$ are thought to be specific states of organisms which, at least sometimes, are the causes of the behavior of those organisms. On the other hand, those of us who are materalistically minded are convinced that those specific mental states are, in some way or other, identical with or realized by some physical (i.e., probably neurophysiological) states of the organisms involved. Or, to put it another way, that there are states of those organisms which have physical as well as mental descriptions or which are physical as well as mental states, and that it is these states which are causally relevant for the production of certain kinds of behavior.

Put this way, the problem of the causal ineffectiveness of mental properties immediately becomes apparent. For now it appears pertinent to ask whether it is the physical or the mental properties of the states in question that are causally relevant for the system's behavior. ${ }^{5}$ Would this behavior take place even if these states lacked all mental proper- 
ties? I think that there is a natural inclination to say that the answer to this question is yes. If an organism is in a certain neurophysiological state it will move certain muscles and thereby produce a certain behavior, quite irrespective of what other properties this state has or lacks. And if the neurophysiological states altered it is quite probable that the behavior produced will also be different. We may feel, however, that this answer is too short. So let us try to become a little clearer on states, state types and their causal relevance for the behavior of a system.

2 .

In an article on the principles of thermodynamics in the Encyclopedia Britannica, 1983 edition (Bd. 18, 291-292) I once happened to read the following remarks on systems and states of systems:

The term system means an identifiable collection of matter that can be separated from everything else by a well-defined surface so that changes in everything need not affect the condition of the collection. . . At any instant of time a system is in a condition called a state, which encompasses all that can be said about the results of any measurements or observations that can be performed on the system at that time. The state at a given instant of time determines the properties of the system. A property is any quantity the value of which depends upon the state but not the history of the system. For a given state the value of a property can be determined by some type of measurement (some physical operation or test). When the values of all properties of two states are identical, the two states are identical; otherwise they are different.

This is very interesting indeed. But it is also, in my view, misleading, not only because of its overly operationalistic flavor, but especially because the distinction between states and properties of a system is not very clear. By this account, it seems, that states are merely the sum of all properties of a system. But this is not a very helpful way to define the state of a system, especially because the fact that the properties of a system are determined by its states is made a trivial consequence of the way states are defined. One reason for the problematic character of the above account is that systems are viewed as wholes without taking into account their constituent parts. And, as I see it, states are best defined in terms of the properties and relations of the parts of a system. So, this is my proposal for a definition of "state": The state of a system is given by the kind of the parts of the 
system, their basic properties and their basic relations to each other. ${ }^{6}$

Now, this sounds pretty vague because what are the parts of a system? Are they only the smallest elementary particles which are contained in the system, or even some higher-order entities which themselves can be said to have parts? And what is meant by the "kind" of a part? Do only physical kinds count? Or are functional kinds such as resistors or transistors admissible, too? I have no clear answer to these questions and I shall not try to find one because nothing of what follows depends on how these questions are answered. At least I hope so.

Another aspect of the vagueness of the proposed definition arises from the double occurrence of "basic" in my proposal. What are the "basic properties" and the "basic relations" of the parts of a system? Obviously, the answer to these questions depends to a certain extent on the answer to the earlier questions. If only elementary particles count as parts, the basic properties of these are likely to be mass, charge, velocity, spin, and other fundamental physical properties. And the basic relations in this case are likely to be the spatial relations of the particles, such as their relative positions, since all other relations will follow from these spatial relations and the properties of the particles, according to the laws of physics. If, on the other hand, even resistors and transistors could be the parts of a system then quite different properties would be relevant. And the most important relation would then be what is connected to what by what means.

It is important to note that states defined in this way are not state tokens but state types. Two different systems, or one system at different times, can have parts of the same kind, which have the same properties and the same relations to each other. So, these systems will be in the same state if we define states according to (5). State tokens, according to this account are instantiations of such states at a time by a particular system. Therefore, saying that two systems are in the same state, I mean to say that they are in different state tokens which, however, are instantiations of the same state as defined in accordance with (5).

Now, what could be meant by saying that states have properties, and that it is perhaps true of some, but not of all of these properties that being in a state which has these properties is causally relevant for a certain effect. As an example, let us take a system $A$ of a number of molecules of an ideal gas contained in a vessel. The state of $A$ - 
according to (5) - is given by the number and kind of the molecules, their basic properties (mass, charge, velocity, etc.), and their relative spatial positions. As is well known, systems like $A$ do not stay in a stable state, but run through a series of states which constantly change, according to the laws of physics. But it is well known too that, at least if $A$ is energetically closed, all these states have a common property, the property of being states in which the molecules involved have the same mean kinetic energy. This is a property of states and therefore defines a certain type of states. So, strictly speaking, individual objects cannot have such properties or belong to such types. But, of course, one can say in such a case that an individual object $x$ is in a state with a certain property, or belonging to a certain type $P$; i.e., for each state type $P$ there is a property $F_{P}$ such that an object has the property $F_{P}$ if and only if it is in a state token which is an instantiation of the state type $P$. So, there is a one-to-one mapping from state properties or state types to corresponding object properties.

Now, what do the states of a system or the properties of these states have to do with the effects of events in which this system is involved? Let us assume that system $A$ has a temperature of $300^{\circ} \mathrm{K}$ so that, if we immerse a mercury thermometer into $A$, the mercury column of this thermometer will rise to the corresponding mark. What is the cause of this event?

We all know that the details of this causal story are rather complicated. When the thermometer is immersed, individual gas molecules continue to collide with the thermometer thereby changing the mean kinetic energy of the molecules of the mercury column. Due to the fact that the mean kinetic energy of these molecules is now greater than before, the mercury expands until, when it reaches the $300^{\circ} \mathrm{K}$ mark, a new equilibrium is achieved.

On the other hand, we also know that the details of this causal story are somewhat irrelevant. In order to raise the mercury column of the thermometer to the $300^{\circ} \mathrm{K}$ mark, it does not matter which molecules hit the thermometer when and at what speed. Rather, the crucial point is that the mean kinetic energy of the molecules of this column increases in such a way that the column itself expands to a certain degree. And this happens whenever the mean kinetic energy of $A$ 's molecules amounts to $6.21 * 10^{-21}$ Joule. So, there obviously is a host of microevents involved in the causation of the macroevent that consists in the rising of the mercury column up to the $300^{\circ} \mathrm{K}$ mark. But ultimately 
these specific microevents are not essential since any other set of microevents that could have occurred under the given conditions would have resulted in the same macroevent. To this extent, it is reasonable to say that it was not the particular states $A$ passed through that were relevant for the rising of the mercury column, but only the fact that all these states shared the property that the mean kinetic energy of the molecules involved amounted to $6.21 * 10^{-21}$ Joule - or, to put it differently, that only one specific property of these states, or one specific property of $A$ (namely the property commonly known as temperature), was causally relevant for this event.

\section{3.}

In the last section I tried to show that, at least in some situations, it is quite reasonable to say that it was a specific property of a state of a system that was causally relevant for an event in the causation of which the system was involved. But I am very sceptical about whether it can be shown along these lines that there are cases in which the property of being in a certain mental state, i.e., a token of a mental state type, is causally relevant for the production of a certain behavior of the system in question.

Let us consider another example. Let $B$ be a system which, being in a certain state $Q$, exhibits the following behavior: it grasps every little ball in its immediate environment. Obviously, $B$ must be equipped with some motor output component (say an extendable arm with a kind of claw) which enables it to pick up small pieces in its environment. And it must also be equipped with some sensory input component that transmits enough information from the environment so that $B$ is able to distinguish balls from other things, and to tell where in its environment a ball is located. ${ }^{7}$

Let us now take a closer look at system $B$, or to be more precise, at the state $Q$ and the behavior $B$ exhibits while being in $Q$. With regard to $Q$, let us assume that $Q$ is a physical state according to (5), i.e., $Q$ is characterized by the physical parts of $B$, the physical properties of these parts and their physical interconnections. $Q$, characterized this way, is certainly a physical state of $B$. On the other hand, this physical state is obviously the basis of a behavioral disposition of $B$, the disposition to grasp and pick up every ball in its immediate environment. Thus, there is at least a temptation to say that a state token of $B$, which 
is an instantiation of the physical state type $Q$, is also an instantiation of a state type which we may call "ball-grasping disposition". And this temptation will become stronger if we find out that there are other state types of this kind which $B$ can assume, e.g. a "cube-grasping disposition"-state type, a "do-nothing disposition"-state type, a "runaway disposition"'state type, etc.

What about the behavior that $B$ exhibits while being in state $Q$ ? It is certainly true that all of $B$ 's behavior in a way consists of bodily movements, i.e., certain specified movements of its arm and claw. But depending on the different sensory inputs, $B$ produces quite different movements in different situations. All these movements, however, have something in common, namely the fact, that they all lead to the result (let us call it $R$ ) that in the end the little ball, wherever it may be, will have been grasped and picked up, i.e., will be in the claw of $B$. So it seems quite clear that we have different descriptions not only for the state $Q$, but also for the behavior produced by $B$ while being in $Q$. $Q$ can be described in terms of $B$ 's physical parts, their properties, and relations and as the disposition to produce a certain kind of behavior, while the output of $B$ can be described in terms of certain physical movements of $B$ 's arm and claw and in terms of ball grasping behavior.

4.

Let us now consider a specific situation where a ball is lying immediately in front of $B . B$, being in state $Q$, does what we expect it to do: it grasps and picks up the ball, i.e., it produces a certain series of movements of its arm and claw which have the effect that the ball is picked up. What are we to say if asked about the cause of this behavior? Or if asked about what states or properties of states of $B$ were causally relevant for the production of this very behavior?

With regard to what has been said about the possibility of double descriptions of $Q$ as well as of the output of $B$, it might seem that the answer to these questions depends on how the output of $B$ is described. If we think of the output of $B$ as bodily movements, it seems obvious that the answer must run: The cause of these movements is the physical state $Q$ and the causally relevant properties are the physical properties of $Q$. If, however, we take the output of $B$ as a ball-grasping behavior, it might, at first glance at least, be tempting to answer these questions in a way similar to the way we treated the case of system $A$ above. 
That is to say, it might seem that in this case too the details of the physical state $Q$ are in a certain sense irrelevant with regard to the production of this behavior. The only thing that matters is that $Q$ instantiates the state type "ball-grasping disposition" since whenever $B$ is in a state which instantiates this state type it will produce ball-grasping movements. ${ }^{8}$

I think, however, that in case of system $B$ this kind of analysis would be entirely inappropriate. In order to be able to see why, it is useful to think of another system $B^{*}$ which is, as we will assume, equipped with the same motor output components and which exhibits, in situations of the same type, the same kind of movements as system $B$. Concerning the physical make-up of the interior of $B^{*}$, however, let us assume that is quite different from that of $B$ so that a fortiori the physical states which produce $B^{*}$ 's behavior are entirely different from the corresponding states of $B$. To have a shorthand, let us assume that $B^{*}$ exhibits ball-grasping movements if and only if it is in a state of type $Q^{*}$ whereas $B$, as we have already assumed, exhibits such movements exactly if it is in a state of type $Q$. So, on the one hand $Q^{*}$ states differ from $Q$-states physically as much as you like. On the other hand, however, $Q^{*}$-states cause in $B^{*}$ the same kind of ball-grasping behavior as $Q$-states in $B$. That is to say, each state token which instantiates either physical state type $Q$ or physical state type $Q^{*}$ also instantiates the higher order state type "ball-grasping disposition". Analysing system $B$ in analogy to system $A$ therefore might lead to the conclusion that, if, on a certain occasion, a certain ball-grasping behavior of $B$ is caused by $B$ 's being in the state token $q$, the relevant property of $q$ is not its belonging to physical state type $Q$, but its belonging to the higher order state type "ball-grasping disposition". And that the same holds if, on another occasion, a certain ball-grasping behavior of $B^{*}$ is caused by $B^{*}$ 's being in the state token $q^{*}$.

This however would mean to overlook a crucial difference. If two states of system $A$ cause the same kind of macroevent because they share the property that the mean kinetic energy of the molecules involved amounts to $6.21 * 10^{-21}$ Joule, these two states may differ in their more specific physical properties, but they nonetheless share some (physical) feature which, as it were, defines a more abstract physical state type. The two state tokens $q$ and $q^{*}$, however, do have nothing in common except that they cause the same kind of movements. And the property to cause a certain kind of movements itself certainly cannot 
be a causally relevant feature with regard to the causation of these movements.

Let us elaborate this crucial point in more detail. If we ask for the causal relevant properties of the physical state token $p$ of system $A$ that causes the mercury column of an immersed thermometer to expand and to rise to a certain mark, we already noticed that not all physical properties of $p$ are relevant in the same way. The same effect would have occured if some of the molecules of $A$ would have been at different spatial positions or would have had velocities of different direction or magnitude, provided only that these differences did not affect $A$ 's overall property of having a mean kinetic energy of $6.21 * 10^{-21}$ Joule. We may however assume that nothing similar is true with respect to the state token $q$ that, on a certain occasion, causes a certain ballgrasping behavior of $B .{ }^{9}$ That is to say, it may well be that even the slightest change of the positions, properties or relations of the components of $S$ would lead to another behavior of $B$. So, with respect to $q$ each physical detail counts. And the same may be true of the state token $q^{*}$ that, on a certain occasion, causes a certain ball-grasping behavior of $B^{*}$, although the details in these two cases are entirely different. So, there is no reason to believe that $q$ and $q^{*}$ share a common property in virtue of which they cause a certain kind of behavior.

The case of system $A$ can be compared with the case of two objects which in turn are placed on a weighing-machine and which both cause the pointer of the machine to point to the same mark. The objects have a number of different properties, e.g., size, shape, color and mass. Obviously, however, the only property that is causally relevant for the movement of the pointer is mass. For this movement will occur in the same way even if you change one of the other properties of the objects, say, their color or their shape.

Let us now, in order to construct an alternative case, assume that we have a set of objects which differ in shape, color and mass in the following way. There are only cubes and spheres, the objects can be blue, red or green, and they have either a mass of $1 \mathrm{~kg}$ or of $2 \mathrm{~kg}$. Let us further assume that there is a fancy machine $M$ the pointer of which can point to two different marks $X$ and $Y$. If we place one of the objects of the mentioned set in front of $M$ it reacts as follows. It points to the mark $X$ if the object is a red cube with a mass of $1 \mathrm{~kg}$ or if it is a green sphere with a mass of $1 \mathrm{~kg}$ or if it is a blue cube with a mass of $2 \mathrm{~kg}$. 
In all other cases it points to mark $Y$. This behavior of $M$ is due to the fact that $M$ first checks all the properties of the objects in front of it and that it is programmed to react to the outcome of this checks in the specified way.

Let us now consider two objects $a$ and $b$ the first of which is a green sphere with a mass of $1 \mathrm{~kg}$ while the second is a blue cube with a mass of $2 \mathrm{~kg}$. If $a$ and $b$ are placed in front of $M, M$ 's pointer will in both cases point to the mark $X$. What, however, are we to say if asked which of the properties of $a$ and $b$ were causally relevant for this effect. In my view, the answer is obvious. In case of object $a$ it was the property of being a sphere and being green and having a mass of $1 \mathrm{~kg}$ and in the case of object $b$ it was the property of being a cube and being blue and having a mass of $2 \mathrm{~kg}$. That is to say, there is no common property which $a$ and $b$ share and which is causally relevant in both cases. ${ }^{10}$ For the only property that $a$ and $b$ have in common is that they both cause $M$ to point to the mark $X$. And this property, if it is a property at all, obviously cannot be the property that is causally responsible for this behavior of $M$. In my view, the case of the systems $B$ and $B^{*}$ bears an exact resemblance to the case of the two objects $a$ and $b$. That is, the $Q$-states of $B$ do have nothing in common with the $Q^{*}$-states of $B^{*}$ except that both states cause a certain behavior. And this property cannot be the causally relevant property with regard to this behavior.

This, in fact, is just the reverse of the coin that what the $Q$-states of $B$ and the $Q^{*}$-states of $B^{*}$ have in common is that both instantiate a dispositional state type, namely the state type "ball-grasping disposition". That belonging to this state type cannot be a property which is causally relevant for the production of the corresponding ball-grasping behavior can also be shown by a more general consideration about the causal relevance of dispositional state types.

As already noted, $B$, being in a $Q$-state, has a certain dispositional property, namely the property to grasp and pick up every ball in its immediate environment. And the same is true of $B^{*}$ being in a $Q^{*}$ state. But it is not only that $B$ has this dispositional property whenever it is in a $Q$-state. It has this dispositional property in virtue of its being in a $Q$-state, in the same way in which $B^{*}$ has the same dispositional property in virtue of its being in a $Q^{*}$-state. In other words: This dispositional property is realized in $B$ by its being in a $Q$-state and in $B^{*}$ by its being in a $Q^{*}$-state. What is important, however, is to see 
what is implied by this, i.e., to see what is implied by saying that a dispositional property is realized by a physical property or state type.

Dispositional properties are generally characterized by the fact that objects which have these properties behave in a characteristic way under certain circumstances. A piece of sugar is watersoluble if it dissolves when put in water. An iron bar is magnetic if it attracts ironfilings and induces a current in a closed circuit. A fluid is an acid if it has a specific sour taste, changes the color of blue litmus paper into red and reacts with certain metals. To say that in a system $S$ a dispositional property $F$ is realized by a certain physical property or state type $P$ is, therefore, to say that it can be shown that in $S P$ is responsible for the behavior that is characteristic of $F .^{11}$ In other words, the dispositional property $F$ can only be realized by the physical property or state type $P$ if it is $P$ that is causally relevant for the behavior in question. Whenever a dispositional property $F$ is realized by a physical property or state type $P$ in a system $S$ and $S$ behaves in a way characteristic of $F$, the causally relevant property or state type for this behavior must be $P$. For otherwise $P$ would not count as an realization of $F$.

To sum up. On the one hand, we have seen that the physical state types $Q$ and $Q^{*}$ have nothing in common except that they both cause a certain kind of behavior. And this property, certainly, cannot be a causally relevant property for the production of this behavior itself. On the other hand, it is exactly due to the fact that $Q$-states and $Q^{*}$-states both cause ball-grasping behavior that all tokens of the state types $Q$ and $Q^{*}$ also instantiate the state type "ball-grasping disposition". Again, however, this property cannot be causally relevant for the particular ball-graspings of $B$ and $B^{*}$. For this property is realized in $B$ by $Q$-states and in $B^{*}$ by $Q^{*}$-states, and these states can realize the dispositional property in question only if it is they which are causally responsible for production of the behavior in question. From whatever angle you look at it, the result is always the same: It is the physical and only the physical properties of $Q$-states and $Q^{*}$-states which are causally relevant with regard to the behavior which is to be explained.

5 .

So far I tried to show that the dispositional property shared by $B$ and $B^{*}$, or the dispositional state type instantiated by all tokens of state 
type $Q$ as well as by all tokens of state type $Q^{*}$, is not a causally relevant property or state type, or, to put it the other way round, that it is only the physical properties of $Q$-states and $Q^{*}$-states which count. But does this mean that the same holds in the case of those much more complex systems which have states that really are tokens of mental state types, such as believing-that- $p$ or wanting-that- $q$ ? One might think that systems $B$ and $B^{*}$ are much too simple to draw such far-reaching conclusions from an analysis of their structure and behavior.

But this is not so, since, although systems with genuine mental states must indeed be more complex by several orders of magnitude, the relation between the physical and the mental properties of the states of these systems, or, to be more precise, the relation between the physical properties of some of their states and the fact that these states are also tokens of certain mental state types, is very similar to the relation between the physical properties of state types like $Q$ and the fact that tokens of this state type are also tokens of the state type "ballgrasping disposition".

This, at least, is true if some kind of functionalism is the correct account of mental states. ${ }^{12}$ Let us take any system $C$ with genuine mental states $M_{1}, M_{2}, \ldots, M_{n}, \ldots$ Then if functionalism is true, there must be physical states $P_{1}, P_{2}, \ldots, P_{n}, \ldots$ of $C$ such that all tokens of $P_{1}$ are also tokens of $M_{1}$, that all tokens of $P_{2}$ are also tokens of $M_{2}$, etc. But what is it that makes the tokens of $P_{1}$ also tokens of $M_{1}$ and the tokens of $P_{2}$ also tokens of $M_{2}$ ? The answer that functionalism gives to this question is well known. The tokens of the physical states of $C$ are also tokens of certain mental states if there is a pattern of causal relations that hold between the physical states $P_{1}, P_{2}, \ldots, P_{n}, \ldots$ and the inputs to system $C$ and the behavior of the system. But this implies, among other things, that the physical states $P_{1}, P_{2}, \ldots, P_{n}, \ldots$ realize the corresponding mental states only if it is they which are causally responsible for the behavior of $C$. In this respect, mental states are not so different from much simpler dispositional states. If they are realized by physical states, it must be these physical states that are causally relevant with regard to the behavior of the systems in question.

This conclusion is strengthened by the observation that the different physical states by which the same mental state is realized in different systems need not have anything in common except that, in a net of inputs, outputs, and other states, they play the same causal role. And, again, I think it is obvious that playing a certain causal role cannot 
itself be a causally relevant property. Thus, there is no important difference between systems like $B$ and systems like $C$. If we take a particular behavior of system $C$ and ask for the causally relevant properties of the states of $C$ that caused this behavior, the answer must be that it is only the physical properties of these states that count.

6.

Does all this prove that the mental is causally inert? Well, that depends. For, first, all that has been said is entirely compatible with the two main tenets of intentional realism:

1. There are intentional states.

2. These states are causally efficacious.

At least, this is true if we understand 1. in the sense of

$1^{\prime}$. There are state tokens that are tokens of mental state types.

In other words, there is at least one interpretation in which the counterfactual

(6) $C$ would not have taken an umbrella if it had not believed that it would rain

may be true, namely the interpretation

(6') $\quad C$ would not have taken an umbrella if it had not been in the state which actually was the belief that it would rain.

But, as already mentioned at the outset, some authors are not so much interested in the causal relevance of mental states as in the causal relevance of mental properties, i.e., in the question whether it is really the mental properties of the states that cause a certain behavior that are causally relevant. With regard to this question, the foregoing considerations were meant to show that the answer must be no. Mental properties cannot be causally relevant as long as they are realized by physical properties. For a physical property can realize a mental property only if $i t$ has a certain causal role, i.e., if it is the physical property which is the causally relevant property with regard to the relevant causal relations.

May be, however, that this conclusion will loose some of its shockingness if one realizes that the considerations which lead to it imply 
the causal irrelevance of a lot of other properties, too. For it is obvious that, according to these considerations, all dispositional and moreover all functionally defined properties must be causally irrelevant. So, many physical properties must also be reckoned among this group. Energy, for example, is a probable candidate, and perhaps even temperature will share the same fate. But this, I think, is something one cannot avoid to accept.

\section{NOTES}

1 An earlier version of this paper has been read at a conference on Mental Causation which was held on March 12-14, 1990, at the Center for Interdisciplinary Research (ZiF), University of Bielefeld, as an integral part of the work of the research group Mind and Brain. I am very grateful to $\mathrm{ZiF}$ for the financial support that made it possible for me to take part in the research group. Thanks also to the organizers of the conference Peter Bieri and Jaegwon Kim.

2 The literature on this question is already vast. Cf. e.g. Honderich $(1982 ; 1983)$, Sosa (1984), Kim (1984; 1989), Stoutland (1985), Johnston (1985), LePore/Loewer (1987; 1989), Dretske (1988a; 1988b), McLaughlin (1989), Horgan (1989), Fodor (1989).

${ }^{3}$ For the formulation of these three theses cf. LePore/Loewer (1987, p. 631).

4 Examples like the following have been advanced especially by Dretske. Cf. e.g. (1988a, p. 32).

5 It is interesting to see that the question here seems to be not what properties or states of the organisms are causally relevant, but what properties of specific states of these organisms are causally relevant.

6 Being in a certain state, therefore, is best understood as having a mereologically defined property. The general schema for the definitions of the corresponding predicates then is:

System $x$ is in state $S$ iff the constituents of $x$ are of such-and-such kind, have such-and-such basic properties and stand in such-and-such basic relations to each other.

7 If someone would like to have a more vivid idea of what system B looks like, there is an impressive picture in Churchland (1986, p. 284).

${ }^{8}$ This seems to be part of the reasoning of LePore and Loewer in (1987).

${ }^{9}$ Of course, it may be that even in the case of system $B$ not all physical properties of $q$ are causally relevant for the causation of the movements of $B$ 's arm and claw. But in this case we could focus our attention on a more abstract physical state type $Q^{\prime}$ instead on the most determinate state type $Q$. On the other hand, it seems obvious to me that the ball-grasping behavior of $B$ just consists in the movements of its arm and claw. Changing details of the make-up of $B$ would result in movements which could no longer be counted as ball-grasping behavior.

${ }^{10}$ In effect, this kind of argument amounts to an outright denial of the claim that there are disjunctive properties. And this, I think, is indeed reasonable to deny. At least, if one understands "property" in a robust and not only in a pleonastic sense. (For a very 
illuminating account of this distinction cf. Schiffer (1987).) In (1990, 20f.) Kim explicitly addresses the question whether it is reasonable to assume that there are disjunctive properties. He concedes that there are strong arguments against this assumption. One such argument starts from the realization, that resemblance is the core of the concept of a property. But, so the argument goes on, there is no sense in which it is reasonable to say that all objects with the alleged property of being round or red resemble each other whereas it is perfectly reasonable to say that all round objects resemble one another in one respect and that all red objects resemble one another in another respect. Kim, however, is not convinced:

I do not find these arguments compelling. It isn't at all obvious that we must be bound by such a narrow and restrictive conception of what nomic properties, or properties in general, must be in the present context. When reduction is at issue, we are talking about theories, theories couched in their distinctive theoretical vocabularies. And it seems that we allow, and ought to allow, freedom to combine, and recombine, the basic theoretical predicates and functors by the usual logical and mathematical operations available in the underlying language, without checking each step with something like the resemblance criterion; that would work havoc with free and creative scientific theorizing. What, after all, is the point of having these logical operations on predicates? When we discuss the definitional reducibility of, say, ethical terms in naturalistic terms, it would be absurd to disallow definitions that make use of disjunctions, negations, and what have you; why should we deny ourselves the use of these operations in forming reductive bridges of other sorts? Moreover, it may well be that when an artificial-looking predicate proves useful, or essential, in a fecund and well corroborated theory and gets entrenched, we will come to think of it as expressing a robust property, an important respect in which objects and events can resemble each other. In certain situations, that recognizing something as a genuine property would make reduction possible may itself be a compelling reason for doing so! $(1990,21)$

In my view, the weak point of this argumentation rests in the last two sentences of the quotation. For it certainly is beyond all question that in introducing new predicates we may use whatever logical and mathematical operations are available. And, what is more, there is no reason to doubt that, by using these operations, we often arrive at very useful and even projectible predicates. But, from all this it does not follow that there must also exist corresponding robust properties. For it may well be that two different objects that both fall under the same predicate and, therefore, both instantiate a certain set of laws, do so in virtue of very different properties. Thus, it may well be, that when a new "predicate proves useful, or essential, in a fecund and well-corroborated theory and gets entrenched", we tend to think of it "as expressing a robust property". But this is no more than a psychological fact. There is no valid rule of inference from the one fact to the other.

${ }^{11}$ For more details of my account of realization, cf. Beckermann (1992a; 1992b).

12 I think, it must also be true if token physicalism is true. For in my view a mental event can be identical with a physical event only if the particular event in question which instantiates a mental event type also instantiates a physical event type by which the mental event type is realized in this case. 


\section{REFERENCES}

Beckermann, A.: 1992a, 'Introduction - Reductive and Nonreductive Physicalism'. in A. Beckermann, H. Flohr and J. Kim (eds.).

Beckermann, A.: 1992b, 'Supervenience, Emergence, and Reduction', in A. Beckermann, H. Flohr and J. Kim (eds.).

Beckermann, A., Flohr, H. and Kim, J. (eds.): 1992, Emergence or Reduction? Essays on the Prospects of Nonreductive Physicalism, Walter de Gruyter, Berlin/New York.

Churchland, P. M.: 1986, 'Some Reductive Strategies in Cognitive Neurobiology', Mind 95, 279-309.

Davidson, D.: 1970, 'Mental Events', in L. Foster and J. W. Swanson (eds.), Experience and Theory. Amherst. Reprinted in: D. Davidson, Essays on Actions and Events, Clarendon Press, Oxford, 1980, pp. 207-225.

Dretske, F.: 1988a, 'The Explanatory Role of Content', in R. H. Grimm and D. D. Merrill (eds.), Contents of Thought, University of Arizona Press, Tucson, pp. 31-43.

Dretske, F.: 1988b, Explaining Behavior, MIT Press, Cambridge, Mass.

Fodor, J.: 1989, 'Making Mind Matter More', Philosophical Topics 17, 59-79.

Honderich, T.: 1982, 'The Argument for Anomalous Monism', Analysis 42, 59-64.

Honderich, T. 1983, 'Anomalous Monism: A Reply to Smith', Analysis 43, 147-149.

Horgan, T.: 1989, 'Mental Quausation'. Philosophical Perspectives 3, 47-76.

Johnston, M.: 1985, 'Why Having a Mind Matters', in E. LePore and B. McLaughlin (eds.), pp. 408-426.

Kim, J.: 1984. 'Epiphenomenal and Supervenient Causation', Midwest Studies 9. 257270.

Kim, J.: 1989, 'Mechanism, Purpose, and Explanatory Exclusion'. Philosophical Perspectives 3, 77-108.

Kim, J.: 1990, 'Supervenience as a Philosophical Concept'. Metaphilosophy 21, 1-27.

LePore, E. and Loewer, B.: 1987. 'Mind Matters', Journal of Philosophy 84, 630-642.

LePore, E. and Loewer, B.: 1989, 'More on Making Mind Matter', Philosophical Topics 17, $175-191$.

LePore, E. and McLaughlin, B. (eds.): 1985. Actions and Events: Perspectives on the Philosophy of Donald Davidson, Basil Blackwell, Oxford.

McLaughlin, B.: 1989, 'Type Epiphenomenalism. Type Dualism, and the Causal Priority of the Physical, Philosophical Perspectives 3, 109-136.

Schiffer, S.: 1987, Remnants of Meaning, MIT Press, Cambridge, Mass.

Sosa, E.: 1984, 'Mind-Body Interaction and Supervenient Causation', Midwest Studies in Philosophy 9. 271-282.

Stoutland, F.: 1985. 'Davidson on Intentional Behavior', in E. LePore and B. McLaughlin (eds.). pp. 44-59.

Manuscript submitted June 20, 1990

Final version received August 19, 1991

Philosophisches Seminar

Georg-August-Universität

Platz der Göttinger Sieben 5

3400 Göttingen

Germany 